\title{
Usuarios finales representantes y desarrollo e implementación de sistemas de información
}

\author{
Calderón-Amaya, José Luis* \\ Rodríguez-Monroy, Carlos** \\ Chaparro-Peláez, Julián***
}

\section{Resumen}

A fin de traer a la luz y argumentar un riesgo crítico, este trabajo persigue evaluar el impacto de ciertos factores actitudinales y conductuales de los usuarios finales representantes en el éxito de los sistemas de información, durante su desarrollo y luego de implementados. Igualmente, se pretende evaluar la posible relación de los usuarios finales representantes con las usuales desviaciones encontradas en estos procesos. La combinación de un método estadístico con un análisis hermenéutico/dialéctico, permitió el logro de los objetivos, al estudiar un macro proyecto del tipo Enterprise Resource Planning. El estudio abarcó el universo de las empresas básicas del aluminio de Venezuela, tomando una muestra no aleatoria de conveniencia del total de desarrolladores de sistemas de información, de usuarios finales representantes líderes y ejecutivos del área de sistemas de información. Los "conocimientos de los usuarios finales representantes" mostraron el mayor impacto y se logró confirmar que el origen de serios rechazos de algunos sistemas de información por los usuarios finales apunta a los mismos usuarios finales representantes. La principal conclusión es que los usuarios finales representantes presentan un serio reto gerencial, en relación directa con pérdidas y fracasos en inversiones en sistemas de información.

Palabras clave: Sistemas de información, desarrollo e implementación, usuarios finales representantes, capacidades y actitudes del usuario final.

Recibido: 20-01-13 Aceptado: 18-01-15

* Doctorando de la Universidad Politécnica de Madrid (UPM), Master in Business Administration por la Colorado State University (CSU), Docente/investigador a tiempo completo de la Universidad Nacional Experimental de Guayana (UNEG). E-mail: jlca1150@gmail.com

** PhD en Organización Industrial, por la UPM, Docente a tiempo completo de la UPM. E-mail: crmonroy@etsii.upm.es

*** PhD en Ingeniería de Comunicaciones, por la UPM, Docente a tiempo completo de la UPM. Email: julian.chaparro@upm.es 


\title{
End-user Representatives and the Development and Implementation of Information Systems
}

\begin{abstract}
In order to bring to light and debate a critical risk, this study seeks to evaluate the impact of certain aptitudinal and behavioral factors of End User Representatives (EUR) on the success of Information Systems (IS) during their development and implementation. Likewise, it intends to assess the possible relationship between EURs and the usual deviations found in these processes. The combination of a statistical method with a hermeneutic/dialectic analysis permitted achieving the objectives through studying a macro project of the Enterprise Resource Planning (ERP) type. This study included all the basic enterprises that comprise the public Venezuelan aluminum sector, taking a nonrandom convenience sample from the population of IS developers, EUR leaders and IS executives. The "EUR knowledge" variable showed the highest impact and it could be confirmed that the origins of some serious IS rejections by end users point to the EUR themselves. The main conclusion is that the EURs pose a serious management challenge in direct relation to losses and failures in IS investments.
\end{abstract}

Key words: Information systems, development and implementation, end-user representatives, end-user capacities and attitudes.

\section{Introducción}

En referencia al sector público, en el cual se ubica esta investigación, Goldfinch (2007:917) sostiene: "... la mayoría de los desarrollos de sistemas de información son infructuosos. Mientras mayor es el tamaño del desarrollo, mayor es la probabilidad de que no sea exitoso. A pesar de la persistencia de este problema durante décadas y el desembolso de vastas sumas de dinero, esta problemática ha recibido sorprendentemente poca atención por parte de la literatura de la administración pública". No obstante, se estima que esta afirmación es extensible a las organizaciones en general.

Diversos autores citan estadísticas alarmantes sobre fracasos, totales o parciales, y excesos en los presupuestos y tiempo de los Proyectos de Desarrollo de Sistemas de Información (PDSI), lo cual se ha venido manteniendo por décadas, a nivel global y con tendencia a incrementar su número, a medida que los PDSI se incrementan (Laudon y Laudon, 2012; Conboy, 2010; Ouadahi, 2008; Wu y Fang, 2007). Ello conlleva a significativos incrementos en costos o pérdidas para las organizaciones en sus inversiones en tecnologías de información ( $\mathrm{TI}$ ), planteando serios retos a todos los niveles gerenciales y a los informáticos involucrados y traduciéndose en la reducción de estas inversiones.

Aunque el estudio de esta temática comenzó en los años 60 (Bartis y Mitev, 2008), la problemática continúa creciendo lo cual refleja la necesidad de profundizar la investigación (Laudon y Laudon, 2012; Mithas et al, 2012; Conboy, 2010; Meissonier y Houzé, 2010; Al-Ahmad et al, 2009). Además, el problema se magnifica cuando un sistema de información (SI) que involucra a clientes y proveedo- 
res es mal utilizado o rechazado por los Usuarios Finales (UF), pudiendo dañar estas relaciones tan críticas, agregando un costo socio-económico de difícil recuperación. De allí, el énfasis en la gestión gerencial para prevenir estas situaciones.

Laudon y Laudon (2012) definen el término UF como los trabajadores que utilizan los SI para realizar las actividades del negocio, diferenciándolos así del personal de informática, de los usuarios externos (por ejemplo, clientes, proveedores, empresas asociadas) y otros con acceso a los SI. Corresponde, por tanto, a los Usuarios Finales Representantes (UFR) aportar las características y especificaciones funcionales aplicativas (no las tecnológicas) de los SI a ser desarrollados para el uso de todos los UF y efectuar la validación respectiva. No obstante, sólo una muestra de los UF es convocada para cumplir con estas tareas asignadas a los UFR en los PDSI, como representantes del resto de la comunidad usuaria de la organización.

Aunque las dimensiones analizadas sobre los UFR han sido estudiadas anteriormente, se estima que las mismas han sido tratadas superficialmente por la investigación al omitir variables indicadoras relevantes o tratarlas de forma separada. Entre la literatura encontrada, los trabajos de Yu-Chih et al (2010) y Kappelman et al (2006) son de los pocos que hacen hincapié en los conocimientos de los UFR como factor clave en los PDSI. Otros factores, como la posible aceptación de la tecnología, la participación, el dominio de las TI y la aversión al cambio son los más estudiados.

Varios investigadores precisan la relevancia de la problemática y recono- cen la necesidad de ahondar en la misma (Mithas et al, 2012; Yu-Chih et al, 2010; Myers, 2009; Yoruk y Ercan, 2006; Bruque et al, 2004; DeLone y McLean, 2003). En el mejor de los casos, aunque este tema haya sido tratado antes, el hecho de que la problemática aun persista es indicativo de la necesidad de profundizarla usando nuevos enfoques, como el presentado aquí.

En las investigaciones encontradas, el éxito de un SI suele ser medido sólo en uno de dos períodos: 1) el período de desarrollo como tal, donde las métricas utilizadas se orientan en última instancia al logro de las estimaciones presupuestarias de dinero y tiempo, como en las investigaciones de Conboy (2010), Kappelman et al (2006) y Rainer y Hall (2003) y 2) el período de post-instalación, en el cual dichas investigaciones se enfocan usualmente en evaluar el éxito del SI en base a parámetros técnicos de funcionamiento, como en los estudios de Myers (2009), Pan et al (2008), DeLone y McLean (2003). Sin embargo, ninguno de esos estudios hallados conecta ambos períodos para medir el impacto ejercido, invariablemente, en el segundo por las aportaciones, basadas en aptitudes y actitudes, de los UFR durante el primero.

Aun cuando la adquisición de paquetes de software aplicativo de proveedores externos supone una adaptación de parámetros más no un proceso de desarrollo, es bien conocido por los practicantes informáticos que cuando sus módulos o aplicaciones brindan una funcionalidad deficiente respecto a la requerida, o no la contemplan en absoluto, es imperativo recurrir a un proceso de desarrollo parcial o completo. 
En resumen, las siguientes características resaltan la originalidad de este estudio: Primera, abordar el vacío hallado en docenas de artículos que tratan el tema de rechazos, pérdidas o excesos en costos en los PDSI, sin referencia alguna a su posible vinculación con un rol deficiente de los UFR y, menos aún, a su inherente responsabilidad. Esta última es igualmente soslayada e ignorada por las organizaciones, la teoría y la investigación.

Segunda, la inserción de los UF en ambos extremos del modelo propuesto, colocándolos en una relación bidireccional con ellos mismos, lo cual muestra su peso crítico, por una parte como los arquitectos responsables de la calidad funcional de sus SI y, por la otra, como el principal grupo de "stakeholders" de cuya aceptación voluntaria dependerá el éxito verdadero de los SI, una vez implantados.

Tercera, la conexión de los dos períodos mencionados con un énfasis en el éxito funcional de negocio, por encima del éxito técnico. Ello mediante la evaluación de este impacto no sólo durante el desarrollo de los SI sino al implantarlos, siendo en este punto donde realmente se verifica la aceptación, el uso y la utilidad de los mismos.

Dos preguntas clave resumen la investigación: a) ¿en qué grado impactaron el éxito de los SI ciertos factores relativos a capacidades y actitudes de los UFR? y b) ¿cómo influyeron los UFR en las desviaciones, especialmente en el tiempo y lo presupuestado, para dicho PDSI? Para responder estas preguntas se plantearon dos objetivos específicos: a) evaluar, mediante un método estadístico, en qué grado los referidos factores impactaron el desarrollo y éxito de los SI involucrados, y b) evaluar cualitativamente la problemática y desviaciones durante el desarrollo y la implementación de los SI, así como la posible relación entre los UFR y las pérdidas por desbordamiento de los tiempos y presupuestos, que suelen traducirse en fracasos totales o parciales en los PDSI.

De lo anteriormente expuesto, el objetivo principal de esta investigación ha sido evaluar el impacto de ciertos factores aptitudinales y conductuales de los UFR en el éxito de los SI, durante su desarrollo y luego de implementados, así como la posible relación de los UFR con las usuales desviaciones encontradas en estos procesos. Esto permitirá traer a la luz y argumentar un riesgo crítico poco o nada internalizado por las organizaciones ni debidamente tratado por la investigación, al soslayar la calidad de dichos factores y la inherente responsabilidad de estos actores.

Como contribución a la práctica se persigue, primero, aportar a gerentes y practicantes involucrados en PDSI un llamado de alerta y la concientización sobre la problemática tratada y, segundo, aportar teoría mediante sugerencias y guías prácticas en forma de parámetros de evaluación para efectuar acciones tendientes a prever y neutralizar los potenciales riesgos. Un aporte adicional a la teoría es la posible ampliación retrospectiva del modelo revisado de DeLone y McLean (2003) al colocar las variables latentes del modelo propuesto como predictivas de los constructos "calidad de la información" y "calidad del SI", como herramienta técnica. Además se han analizado en mayor amplitud y profundidad ciertas dimen- 
siones críticas de los UFR, las cuales continúan siendo política y socialmente un tabú en las organizaciones.

Sin embargo, no es un objetivo de esta investigación el estigmatizar el rol de los UFR, sino darle visibilidad a una problemática que usualmente afecta a varios niveles de dirección organizacional y es observada en diferentes tipos de organizaciones durante una larga experiencia práctica en PDSI.

\section{Los usuarios finales y el éxito/fracaso de los SI}

Por décadas, los investigadores han propuesto modelos sobre el éxito de los $\mathrm{SI}$, validados empíricamente, incluyendo variables relacionadas con los UF en un intento por entender su rol (Laudon y Laudon, 2012; Al-Ahmad et al, 2009; Myers (2009); Bartis y Mitev, 2008). Sin embargo, este continúa siendo un elemento complejo y amerita mayor investigación sobre su relación con las estadísticas antes citadas. No obstante, la revisión del estado del arte no arrojó estudio alguno sobre el éxito/fracaso de los SI con un abordaje del tema desde la presente perspectiva.

Un estudio de Bruque et al (2004) analizó las relaciones entre algunos recursos basados en factores intangibles humanos y los resultados empresariales, así como la relación entre estos últimos y el grado de uso de las TI. No obstante, reconocieron no haber analizado empíricamente la relación entre esos recursos intangibles humanos y el grado de aceptación de las TI. La presente investigación se centra precisamente en este último análisis, partiendo del uso voluntario y sa- tisfactorio, no forzado de las TI y en las consecuencias de los desempeños deficientes. Como plantean Andrade y Mandrillo (2004:2), los SI y las tecnologías de soporte "se institucionalizan a través del poder, lo cual se explica al examinar la dimensión política de las organizaciones y los elementos que la conforman".

Los SI basados en computadores reafirman las prácticas sociales de las organizaciones. Así, al ser considerados como un conjunto de prácticas sociales automatizadas y recursivas, estos encierran estructuras de poder ocultas en la toma de decisiones para su desarrollo que deben ser analizadas por la gerencia (Andrade y Mandrillo, 2004). También, se sostiene la opinión de Walsham (1995), sobre la creciente criticidad de los aspectos sociales respecto a los $\mathrm{SI}$, especialmente en los últimos años y en lo concerniente a los significados e interpretaciones humanas.

Reconocidos investigadores destacan el impacto del factor humano en la construcción de los $\mathrm{SI}$ y algunos de ellos argumentan la prestación de una atención muy marginal de parte de la literatura sobre el éxito de los $\mathrm{SI}$ a los aspectos conductuales sociales involucrados (Laudon y Laudon, 2012; Mithas et al, 2012; Chen et al, 2011; O'hEocha et al, 2010; Myers, 2009; Kappelman et al, 2006; Llorens, 2005). Igualmente, mantienen la creencia de que tales conexiones continúan aún sin ser bien establecidas y la historia de las competencias sigue estando poco explorada (Yu-Chih, et al, 2010). Se comparte el criterio de Andrade (2003) respecto a visualizar los SI como herramientas legitimadoras del poder en las organizaciones, por lo cual en este estudio se justifica aún más los enfoques utiliza- 
Usuarios finales representantes y desarrollo e implementación de sistemas... Calderón-Amaya, José Luis, Rodríguez-Monroy, Carlos y Chaparro-Peláez, Julián

dos, complementando un análisis estadístico con un análisis interpretativo, mediante la aplicación del método conocido como mixto o triangulación.

\subsection{Estimación del éxito de los $\mathrm{SI}$}

Para la estimación del éxito de los $\mathrm{SI}$, este estudio se basa inicialmente en el modelo revisado de DeLone y McLean (2003), el cual para el año 2003 ya había sido probado empíricamente en cerca de 300 trabajos en revistas científicas arbitradas, desde su aparición en 1992. Este modelo coloca la dimensión "satisfacción de los UF" como variable criterio o dependiente de tres variables latentes, analizadas post-mortem, de todo SI como son la "calidad de la información" generada, la "calidad del sistema", como herramienta tecnológica, y la "calidad de los servicios recibidos" del área de informática.

Igualmente, se halla justificación en otros estudios los cuales también sostienen el predominio de la satisfacción de los UF sobre la de otros interesados o stakeholders y como sinónimo del éxito de un SI (Conboy, 2010; Yu-Chih et al, 2010). Por ejemplo, Ravichandran y Rai (2000:132) consideran esta dimensión como crítica y por tanto "... es considerada una medida válida de la calidad de los sistemas de información, ya que refleja la evaluación subjetiva de sus características y su funcionalidad. Para Yoruk y Ercan (2006), el desarrollo de un SI implica un proceso costoso que generalmente no cumple con las expectativas de los UF tras su implementación.

Yoruk y Ercan (2006), también resaltan una situación común en la cual profesionales de la informática, diseñadores y programadores, a menudo tienen dificultades para comprender los requerimientos de los UF y que más de un $50 \%$ de los errores durante el proceso de desarrollo tienen lugar en la etapa de definición de requerimientos de la fase de análisis. Dada la criticidad de los pasos del diseño y la programación de un SI, estos errores dan lugar a retrasos por reprocesos, desborde del presupuesto y, eventualmente, insatisfacción con el producto final, conduciendo usualmente a su rechazo.

Habiendo estudiado una muestra de más de 400 UF de SI tipo Enterprise Resource Planning (ERP), Somers et al (2003) concluyen que la medida de la satisfacción de los UF es quizás el factor más determinante para la estimación del éxito de un SI. Esto es soportado también por Hsu et al (2006). Por tratarse de percepciones humanas, el éxito para algunos interesados puede significar un fracaso para otros (Al-Ahmad et al, 2009). El estudio de Myers (2009), analizó el desarrollo e implementación de un SI de nómina realizado por decisión del Departamento de Educación de Nueva Zelanda; el cual, a pesar de haber sido instalado y declarado exitoso por los demás stakeholders al considerar su funcionamiento aceptable, debió ser descontinuado ante el rechazo de los UF.

Esto último tiende a reforzar la tesis que son los UF quienes usualmente determinan la aceptación o rechazo de un SI y ello ocurre con mayor frecuencia cuando esta actitud es promovida por supervisores o colegas en el entorno laboral. Los Modelos de Aceptación de la Tecnología (TAM, por las siglas en inglés de Technology Acceptance Model), TAM, TAM2, 
TAM3 y otros similares, son de utilidad pues analizan ciertas variables conductuales condicionantes de la aceptación y uso de las TI (Ramírez-Correa et al, 2010). Sin embargo, se ha demostrado la invalidez de que la tecnología es la impulsora del cambio (Myers, 2009).

\subsection{Conocimientos aportados por los UFR}

En el modelo propuesto, la dimensión "conocimientos de los UFR" se refiere a aquellos relacionados con aspectos estratégicos, funcionales, administrativos y socio-culturales del negocio y del área usuaria en cuestión, presentes en el conocimiento tanto explícito como tácito (Nonaka, 1991; Schultze y Leidner, 2002). La presencia de incertidumbre en los UFR acerca de los requisitos de información a ser aportados tiene un impacto directo y negativo sobre la capacidad de respuesta de los SI en desarrollo (ShihChieh, et al, 2008). Esta situación se acentúa debido a velocidad de cambio en los entornos de negocio y a la evolución de los procesos organizativos (Kautz et al, 2010). Bruque et al (2004) se preguntaron si las $\mathrm{Tl}$ en las organizaciones eran mejor aceptadas y utilizadas cuando las capacidades humanas y de gestión complementarias eran desarrolladas simultáneamente, pero no lograron analizarla desde un enfoque empírico.

El presente estudio argumenta que el conocimiento de las estrategias y planes del negocio debe provenir de la Alta Gerencia y las unidades funcionales involucradas; no de los informáticos, cuya misión se basa en el conocimiento, el domi- nio y la aplicación de las complejas y cambiantes herramientas de TI. En la práctica se ha observado que aun cuando los informáticos posean este conocimiento no es de su competencia el dictar pautas sobre los mismos, evitando así iniciativas erróneas. Lo anterior conduce a plantear la primera hipótesis:

- H1: El éxito de un SI, durante su desarrollo y principalmente una vez implementado, está altamente correlacionado con el conocimiento aportado por los UFR.

\subsection{Participación de los UFR}

La noción del constructo "participación de los UFR" ha sido estructurada para evitar limitarlo a lo que refiere la cita popular "... participación de cuerpo presente pero de mente ausente." Así, este constructo apunta a una participación oportuna, relevante y de actitud proactiva traducible en una contribución significativa (Markus y Mao, 2004; Barki et al, 2001; Barki y Hartwick 1989). Este factor comprende las actividades realizadas por los UFR al representar a sus iguales ausentes en un PDSI (la inmensa mayoría) a fin de prevenir la insatisfacción con el nuevo SI por no cumplir con sus requerimientos de información, característica principal de todo SI (Terry y Standing, 2004).

Sólo una participación de calidad puede llevar a la correspondencia entre las entregas del SI y las necesidades de los UF a ser satisfechas (Saarinen y Saaksjarvi, 1990). Es la calidad de la participación, más que su frecuencia, lo relevante. Esto lleva a plantear la hipótesis siguiente: 
- H2: El éxito de un SI, durante su desarrollo y principalmente una vez implementado, está altamente correlacionado con la participación aportada por los UFR.

\subsection{Compromiso de los UFR}

El constructo "compromiso de los UFR" es tratado separadamente del constructo "participación de los UFR" por Barki y Hartwick (1989) para referirse a un estado psicológico subjetivo que refleja la significación y respaldo personal dado por los UFR a un PDSI. Una condición necesaria para conseguir un compromiso efectivo es la adopción de estructuras organizacionales orgánicas, caracterizadas por la flexibilidad y el dinamismo derivadas de la toma de decisiones descentralizadas (Rastrollo y Castillo, 2004). De esta manera, se puede mantener un buen grado de formalización permitiendo crear interacciones para ayudar incluso a capitalizar el conocimiento, transformándolo de tácito a explícito.

Afirman Rastrollo y Castillo (2004:141): "la formalización del comportamiento individual se logra con la internalización de normas y valores, antes que mediante la imposición de reglas de conducta". Básicamente es un tema de naturaleza humana y, como lo plantea la "Escuela de Aprendizaje Situado"; los UFR se comprometen con un PDSI "sólo cuando su ámbito de trabajo es directamente afectado." (Rastrollo y Castillo (2004: 517). También García y Rangel (2001) en su estudio empírico sobre la implementación reciente de un SI ERP para los Departamentos de Recursos Humanos de seis organizaciones encontraron que en la mitad de dichas empresas la medida promedio de resistencia al cambio, como elemento degradante del compromiso de los UFR, se hallaba entre los niveles mediano y alto. Esto lleva a siguiente hipótesis:

- H3: El éxito de un SI, durante su desarrollo y principalmente una vez implementado, está altamente correlacionado con el compromiso aportado por los UFR.

En estos dos últimos factores, referidos a la participación y el compromiso, se inserta la responsabilidad de los UFR por el éxito/fracaso de sus $\mathrm{SI}$, el cual pareciera tan tabú como el término "poder" en las organizaciones. Citan Andrade y Mandrillo (2004:12) el hallazgo de Kotler quien luego de analizar 2.000 artículos en el Harvard Business Review, correspondientes a un período de 20 años, sólo 5 de ellos incluían en sus títulos la palabra "poder", tratando el tema como un "secreto sucio de familia: todo el mundo lo conoce, pero nadie se atreve a discutirlo abiertamente" (Andrade y Mandrillo, 2004:612). Incluso, apuntan Andrade y Mandrillo (2004:612), quienes referencian a Kotler que en un examen más reciente, halló el término "poder" en los títulos de apenas cerca de una docena de un total de 3.000 artículos publicados entre 1960 y mediados de 1999. Quizás por ello el "poder" de los UFR respecto al tema tratado sea también otro secreto de familia, por lo cual es soslayado por la teoría gerencial y la investigación. 


\subsection{Beneficios esperados}

La dimensión "beneficios esperados" es adoptada del modelo de DeLone y McLean (2003) y está representada por un aumento en el rendimiento laboral individual y grupal de los UF, en función de la manera como estos perciben que un SI satisface sus necesidades laborales. En su modelo, esta dimensión puede verse como variable mediadora entre la satisfacción de los UF y los beneficios esperados por el resto de stakeholders en la organización, resumidos en mayores ingresos o reducción de costes (Mithas et al, 2012; Byrd et al, 2006; DeLone y McLean, 2003), estando esta última dimensión fuera del alcance de esta investigación.

Davis et al (1989) afirmaron hace más de dos décadas que ningún beneficio podrá derivarse de los SI cuando no son aceptados y utilizados efectivamente al ser percibidos por los UF como herramientas útiles para recibir y procesar la información necesaria para el desempeño eficaz en sus labores. Esto permite a presentar la hipótesis siguiente:

- H4: Los beneficios esperados de un SI por los UF están altamente correlacionados con su propia satisfacción con el mismo, una vez implementado.

Esta investigación conecta dos categorías distintas y separadas de resultados mencionadas por Markus y Mao (2004:525): "El éxito del desarrollo de un sistema y el éxito de su implementación". Esto coincide con Sawyer (2001, citado por Wagner y Newell, 2007:509) cuando dice: "Es sólo durante la instalación cuando los usuarios se involucran profunda- mente por primera vez en la evaluación de cómo el software satisface sus necesidades", opinión asumida como verdad en esta investigación. No obstante la importancia de la primera categoría, sus parámetros sólo se asocian a la eficiencia alcanzada en el proceso de desarrollo, no a la efectividad del producto creado. La segunda, sí apunta a la utilidad real percibida en función de los beneficios derivados del SI. El modelo a validar se ilustra en la Figura 1 y la división entre ambas categorías -y períodos- es señalada mediante una línea vertical discontinua.

\section{Necesidad de complementar enfoques cuantitativos y cualitativos}

En esta sección, los enfoques cuantitativos y cualitativos se combinan desde el punto de vista metodológico, para reforzar la validez de los resultados obtenidos. Sin embargo, se incluye un breve soporte teórico a su aplicación.

La población estudiada abarcó las cuatro grandes empresas industriales del sector del aluminio de la República Bolivariana de Venezuela. Dichas industrias, en conjunto con las del hierro, el acero y el oro son las principales empresas básicas no petroleras de la nación. Las mismas fueron uniformadas bajo un mismo macro PDSI para la instalación del $S A P / R 3$, un paquete de SI integrados del tipo ERP y donde los UFR tuvieron un rol protagónico, para la adaptación e instalación de los diferentes SI o módulos del SAP/R3, así como el desarrollo dentro del mismo de la funcionalidad requerida no incluida en este paquete. La importancia 
Usuarios finales representantes y desarrollo e implementación de sistemas... Calderón-Amaya, José Luis, Rodríguez-Monroy, Carlos y Chaparro-Peláez, Julián

Figura 1

\section{Usuarios finales representantes y satisfacción de usuarios finales en el desarrollo e implementación de sistemas de información: \\ Modelo propuesto \\ $[$ Producto $=$ SI $]$}

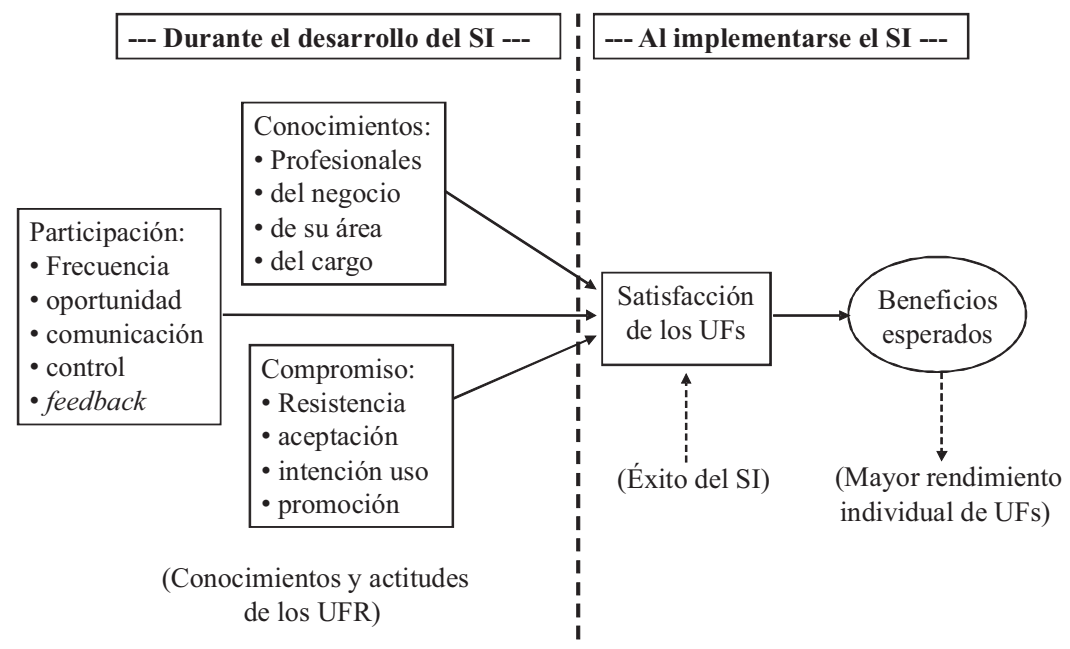

SI: Sistema de Información

UFs: Usuarios Finales

UFR: Usuarios Finales Representantes

Fuente: Elaboración propia.

de estas empresas, la variedad y la complejidad del PDSI y los procesos involucrados fueron aspectos relevantes para su elección.

El trabajo de campo se basó en una muestra de unas 200 unidades informantes, siendo la mayoría profesionales con vasta experiencia, como ejecutivos, desarrolladores de SI y UFR líderes del proyecto SAP/R3, obtenida bajo la técnica de muestreo no aleatorio de conveniencia, usada por investigadores como RamírezCorrea et al (2010). Se utilizaron las técnicas de la encuesta y la entrevista, durante un lapso total de recolección de datos aproximado de seis meses, culminado a mediados del año 2011. Siendo los infor- máticos quienes experimentan de manera directa y en mayor grado la problemática analizada, se estudió la misma básicamente desde su perspectiva.

En concordancia con la teoría sobre el tema (Myers, 2009), se aplicó un doble enfoque analítico estadístico-interpretativo, utilizando la técnica de triangulación, definida como la combinación de diferentes métodos de investigación. Para recolectar los datos primarios a ser analizados cuantitativamente se utilizó un cuestionario con escala tipo Likert de amplitud cinco. Igualmente, se utilizó un instrumento tipo entrevista semi-estructurada para el análisis interpretativo, incluyendo recolección de datos sobre las 
mismas dimensiones y variables presentes en el cuestionario (Cuadro 1).

Una posible crítica a este punto pudiera ser el énfasis en las percepciones de líderes informáticos quienes opinaron sobre sus experiencias con los factores cualitativos y cuantitativos referidos a los UFR convocados a este macro PDSI. Además de ser ellos quienes perciben la problemática de primera mano, se estima que los juicios emitidos por profesionales como estos, con una extensa trayectoria en PDSI y trabajando en interacción constante con los UFR, suelen tener un alto grado de confiabilidad. Por ello, esta misma estrategia ha sido utilizada efectivamente por diversos investigadores (Conboy, 2010; O'hEocha et al, 2010; Yu-Chih et al, 2010; Bartis y Mitev, 2008; ShihChieh, 2008; Subramanian et al, 2007).

Una razón de peso para lo anterior radica en la tendencia de los UF, por di-

\section{Cuadro 1}

\section{Aspectos directamente relacionados con los usuarios finales representantes, evaluados} mediante la encuesta y la entrevista

\begin{tabular}{|c|c|}
\hline Dimensiones & Variables \\
\hline \multirow{8}{*}{ Conocimientos } & Objetivos de la organización \\
\hline & Objetivos de su área \\
\hline & Políticas de la organización \\
\hline & La cultura organizacional \\
\hline & La organización informal \\
\hline & Información relacionada con su cargo \\
\hline & Capacidad para trabajar en grupo \\
\hline & Capacidad para representar a su comunidad usuaria \\
\hline \multirow{7}{*}{ Compromiso } & Actitud ante el reemplazo de los SI vigentes \\
\hline & Actitud ante el cambio de los procesos vigentes \\
\hline & Expectativas con el nuevo SI respecto al trabajo \\
\hline & Expectativas con el nuevo SI respecto al rendimiento individual \\
\hline & Entendimiento de las razones para el cambio \\
\hline & Apoyo manifiesto al proyecto \\
\hline & Actitud del supervisor del área \\
\hline \multirow{6}{*}{ Participación } & Frecuencia de la participación \\
\hline & Relevancia de las aportaciones \\
\hline & Nivel de disponibilidad \\
\hline & Control de calidad \\
\hline & Feedback \\
\hline & Habilidades comunicacionales \\
\hline
\end{tabular}

Fuente: Elaboración propia. 
versas razones, a ocultar sus errores, algo observado en la práctica y de lo cual también dan fe Straub et al, 1995 citado por DeLone y McLean, 2003), quienes al estudiar 458 UF de un SI de correo de voz encontraron una diferencia significativa entre el uso auto-reportado y el uso registrado automáticamente por el computador. Al comparar las respuestas de los mismos con un contador automático oculto en el SI, se determinó que los UF no dieron información verdadera respecto a su frecuencia de uso del SI. Así, centrar este énfasis en los profesionales informáticos es más confiable que el haber encuestado un mayor número de UF sobre sus propias aptitudes y actitudes.

\subsection{Aproximación para el análisis cuantitativo}

Para validar las métricas, se llevó a cabo una prueba piloto con 12 analistas experimentados en el desarrollo e implementación de SI obteniendo un valor de confiabilidad del Alfa de Cronbach superior a 0,89 . Los datos se sometieron a análisis factorial, con extracción de componentes principales, método de rotación tipo Varimax con Kaiser y un análisis de varianza con criterio de autovalor 1 .

Las dimensiones se configuraron con variables indicadoras tomadas de estudios empíricos similares, como los de Yu-Chih et al, 2010; Lesca y Caron-Fasan, 2008; Ouadahi, 2008; Kappelman et al, 2006; Shih-Chieh et al, 2008; DeLone y McLean, 2003; Saarinen, 1996 y de la literatura sobre el tema (Cuadro 1). Este análisis se basó en un muestreo no aleatorio de conveniencia (Ramírez-Correa et al, 2010), enviando el cuestionario por co- rreo electrónico a cerca de 200 encuestados, quienes han sido miembros activos de este macro PDSI durante todas las fases de desarrollo y de implementación, pudiendo dar testimonio de las variables estudiadas en los dos períodos mostrados en el modelo y sus consecuencias.

Con las respuestas válidas de 107 cuestionarios se efectuó un análisis estadístico mediante la técnica Partial Least Squares (PLS), adecuada para probar nuevos modelos (Wold, 1982) usando muestras pequeñas (Lohmoller, 1989). Se utilizaron indicadores de tipo reflectivo debido a su dependencia de sus respectivas variables independientes, a la alta correlación existente entre ellos y a su no afectación por la multicolinearidad (Haenlein y Kaplan, 2004). La "satisfacción de los UF" es tratada como variable mediadora, por ser un factor capaz de regular el impacto de las variables independientes sobre y la variable dependiente "beneficios esperados" por ellos mismos.

\subsection{Aproximación para el análisis cualitativo}

Se realizó un análisis interpretativo del discurso y del contenido de tipo hermenéutico/dialéctico; aplicando la doble-hermenéutica el investigador "... debe hablar el mismo lenguaje de las personas bajo estudio (o, por lo menos, ser capaz de entender una interpretación o traducción de lo que sea dicho)" (Myers, 2009:190). Luego de décadas de ejercicio profesional en la disciplina de los SI, este requisito no representó obstáculo alguno. También se trabajó en función de los conceptos del círculo hermenéutico y prejuicio (Myers, 2009:185-186). 
Para este enfoque cualitativo se realizaron entrevistas individuales y directas a siete informantes clave: cuatro ejecutivos, quienes estuvieron trabajando activamente durante todo el macro proyecto del SAP, y tres UFR miembros permanentes del equipo de este proyecto, quienes como líderes por las áreas usuarias interactuaron con los demás UFR guiándolos para la adaptación/desarrollo de los módulos del SAP/R3.

La respuesta a la posible pregunta de cómo se puede generalizar a partir de un único caso de estudio como el presente ha sido tomada de Yin (1989), quien sostiene la posibilidad de generalizar a través de proposiciones teóricas. También fueron considerados los cuatro tipos de generalización de Walsham (1995), por no ser mutuamente excluyentes para realizar análisis interpretativos, siendo estos: a) desarrollo de conceptos, b) creación de teoría, c) delineación de consecuencias concretas y d) contribución de percepciones útiles.

Este análisis se centró en una combinación de los dos últimos tipos definidos por Walsham (1995); es decir, en el delineamiento de implicaciones específicas y en la búsqueda de la contribución de una percepción útil a investigadores y practicantes. Entre las principales implicaciones consideradas están las interrelaciones entre cada una de las fases tradicionales de cualquier PDSI (análisis, diseño, programación, pruebas e implementación) con los factores aquí estudiados de los UFR (conocimientos, compromiso y participación).

Resumiendo ambas aproximaciones, la Figura 2 muestra el esquema aplicado.

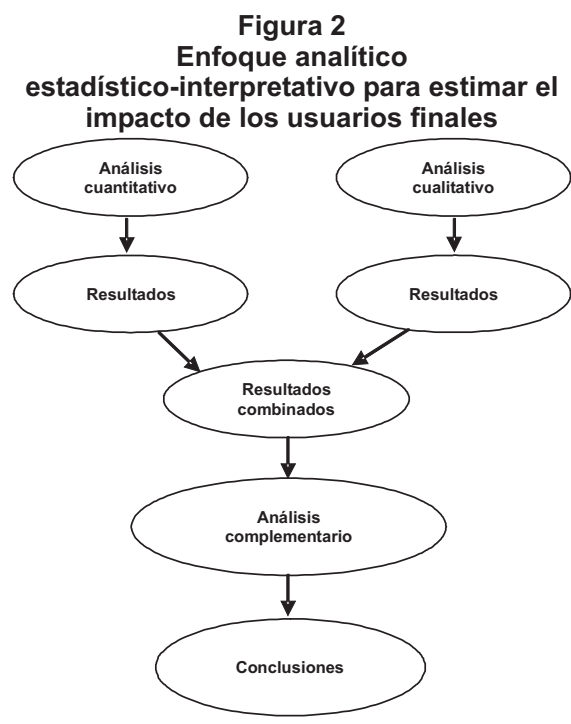

Fuente: Elaboración propia.

\section{El rol de los UFR y la medición de su impacto sobre la satisfacción de los UF}

Un análisis descriptivo reveló que la mayoría de las unidades informantes estaban en el rango entre 31 y 45 años de edad, con educación universitaria, una antigüedad laboral entre 8 y 10 años y una experiencia profesional entre 8 y 12 años. Un 56\% de la muestra estuvo representado por el sexo masculino, permitiendo un equilibrio en la influencia de esta variable demográfica. La encuesta estructurada se basó en un cuestionario con escala Likert de cinco puntos, donde 1 = total desacuerdo, 2 = parcial desacuerdo, 3 = no sé o indeciso, 4 = parcial acuerdo y $5=$ total acuerdo. Al ser las variables indicadoras de tipo ordinal, los valores de la mediana son considerados como los más representativos, siendo el más alto el referido los conocimientos de los UFR (3.413), segui- 
Usuarios finales representantes y desarrollo e implementación de sistemas... Calderón-Amaya, José Luis, Rodríguez-Monroy, Carlos y Chaparro-Peláez, Julián

dos de participación (3.320) y compromiso (3.266). Estos resultados descriptivos confirman el alto nivel de madurez, preparación profesional y experiencia laboral buscada en la muestra.

La confiabilidad de los ítems, de tipo reflectivo, de cada constructo fue determinada mediante las cargas de dichos indicadores utilizando PLS. Se encontraron valores entre 0.778 y 0.892 , considerándose aceptables cargas entre $0.5 \mathrm{y}$ 0.6 (Cepeda y Roldán, 2004), sin eliminar ningún ítem. La validez convergente y la confiabilidad interna de los constructos se establecieron mediante la estimación de la confiabilidad compuesta, observándose valores por encima del umbral de 0.70 , así como valores de la varianza media extraída por encima de 0.50 (Tabla 1 ). Estos valores mínimos de 0.70 y 0.50 se estiman aceptables para la investigación en ciencias sociales (Bacon et al, 1995).

La validez discriminante fue determinada utilizando la Media de la Varianza Extraída (AVE, por las siglas en inglés de Average Variance Extracted). En la Tabla 2 puede observarse como la raíz cuadrada de los valores AVE es mayor a las correlaciones con los demás constructos y por encima de 0.7 .
Las hipótesis fueron evaluadas mediante el examen de los coeficientes de camino $(\beta)$ y sus niveles de significación (se aceptaron $\beta \geq 0,2$ ). Un bootstrapping con 500 sub-muestras fue realizado para comprobar la significación estadística de cada uno de los coeficientes de los caminos. La varianza explicada $\left(R^{2}\right)$ en las variables latentes endógenas y el coeficiente de significación de regresión (Ftest), sirven como indicadores de la capacidad explicativa del modelo. Los path coefficients muestran correlaciones significativas entre las variables predictivas y la variable criterio, así como entre esta última y los beneficios esperados (Tabla 3). Sobre la base en estos resultados, se aceptan las cuatro hipótesis $\mathrm{H} 1$, $\mathrm{H} 2, \mathrm{H} 3 \mathrm{y} \mathrm{H} 4$.

Los resultados del modelo están ilustrados en la Figura 3.

\section{Entendiendo el impacto y consecuencias de los factores analizados}

A continuación, el análisis hermenéutico/dialéctico, enfocado en el ámbito humano y socio-político empresarial, necesario para poner en contexto y lograr

Tabla 1

Usuarios finales representantes y satisfacción de los usuarios finales en el desarrollo e implementación de sistemas de información: Análisis factorial confirmatorio

\begin{tabular}{lcc}
\hline \multicolumn{1}{c}{ Variables } & CC $^{*}$ & AVE \\
\hline Conocimientos de los UFR & 0.892 & 0.541 \\
Participación de los UFR & 0.908 & 0.662 \\
Compromiso de los UFR & 0.778 & 0.504 \\
Satisfacción de los UF & 0.875 & 0.690 \\
Beneficios esperados por los UF & 0.837 & 0.789 \\
\hline
\end{tabular}

CC: Confiabilidad Compuesta. AVE: Varianza Media Extraída. UFR: Usuarios Finales Representantes. UF: Usuarios Finales. Fuente: Elaboración propia. 
Tabla 2

Usuarios finales representantes y satisfacción de los usuarios finales en el desarrollo e implementación de sistemas de información: Validez discriminante

\begin{tabular}{lccccc}
\hline \multicolumn{1}{c}{ Variables } & $\begin{array}{c}\text { Conocimientos } \\
\text { de los UFR }\end{array}$ & $\begin{array}{c}\text { Compromiso } \\
\text { de los UFR }\end{array}$ & $\begin{array}{c}\text { Participación } \\
\text { de los UFR }\end{array}$ & $\begin{array}{c}\text { Satisfacción de } \\
\text { los UF }\end{array}$ & $\begin{array}{c}\text { Beneficios } \\
\text { esperados }\end{array}$ \\
\hline $\begin{array}{l}\text { Conocimientos de } \\
\text { los UFR }\end{array}$ & 0.736 & & & & \\
$\begin{array}{l}\text { Compromiso de } \\
\text { los UFR }\end{array}$ & 0.512 & 0.710 & & & \\
$\begin{array}{l}\text { Participación de } \\
\text { los UFR }\end{array}$ & 0.572 & 0.603 & 0.814 & 0.831 & \\
$\begin{array}{l}\text { Satisfacción de } \\
\text { los UF }\end{array}$ & 0.613 & 0.505 & 0.549 & & 0.888 \\
$\begin{array}{l}\text { Beneficios } \\
\text { esperados }\end{array}$ & 0.579 & 0.649 & 0.672 & 0.733 & \\
\hline
\end{tabular}

NOTA: Los elementos diagonales representan la raíz cuadrada del AVE de la validez discriminante. UFR: Usuarios Finales Representantes. UF: Usuarios Finales.

Fuente: Elaboración propia.

Tabla 3

Usuarios finales representantes y satisfacción de los usuarios finales en el desarrollo e implementación de sistemas de información:

Prueba de las hipótesis

\begin{tabular}{lccc}
\hline \multicolumn{1}{c}{ Hipótesis } & $\begin{array}{c}\text { Coeficientes de ruta } \\
\text { (Path coefficients) }\end{array}$ & t-statistics & Aceptada \\
\hline UFR-conocimientos » Satisfacción & 0.573 & 18,214 & Sí \\
UFR-compromiso » Satisfacción & 0.469 & 11,374 & Sí \\
UFR-participación » Satisfacción & 0.505 & 9,053 & Sí \\
Satisfacción UF » Beneficios esperados & 0.733 & 14,257 & Sí \\
\hline
\end{tabular}

UFR: Usuarios Finales Representantes. UF: Usuarios Finales.

Fuente: Elaboración propia.

entender los resultados estadísticos, arrojó interesantes resultados. El constructo "conocimientos de los UFR" mostró también su mayor relevancia, en especial, durante la definición de las especificaciones sobre la funcionalidad requerida de cada SI, surgiendo problemas serios en los casos donde la calidad de esta dimensión fue baja, por la elección equivocada de los UFR. Las fallas en la "participación de los UFR" se ubicaron mayor- mente en la frecuencia y la oportunidad de la misma, en una alta ocupación laboral, en la comunicación y el feedback, influyendo también el rechazo a algunos cambios.

Respecto a la dimensión "compromiso de los UFR", representada por la evaluación de variables referidas básicamente a la resistencia a modificaciones en los procesos, procedimientos y en la funcionalidad, se evidenció cómo, en al- 
Figura 3

Usuarios finales representantes y satisfacción de los usuarios finales en el desarrollo e implementación de sistemas de información:

Resultados de la evaluación estadística del modelo propuesto

$[$ Producto $=$ SI $]$

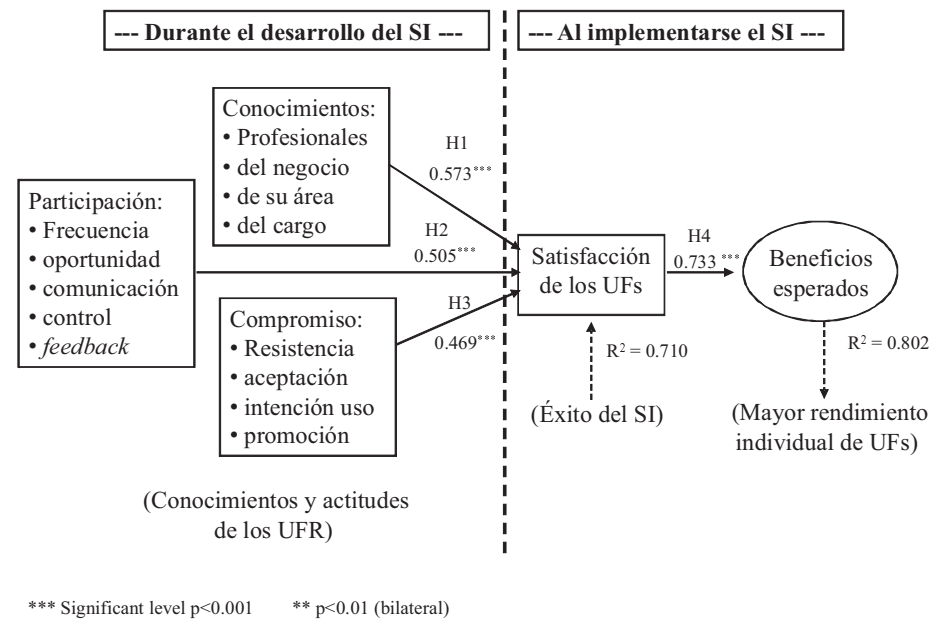

Fuente: Elaboración propia, a partir del análisis estadístico de las encuestas.

gunos casos clave, estos aspectos afectaron en forma significativa el PDSI. Además, se analizó la alta correlación entre la variable "satisfacción de los UF", como variable mediadora, y la variable "beneficios esperados por los UF" ya que la calidad del rol de los UFR impacta la calidad de los SI, afectando los beneficios esperados por los mismos UF. De la calidad y cantidad de estos últimos beneficios dependerán los beneficios esperados por el resto de los stakeholders, cuyo análisis no está incluido en este estudio.

Un claro ejemplo de lo anterior estuvo en los SI de nómina y administración de $\mathrm{RRHH}$, difíciles de automatizar principalmente por las complejidades subyacentes en las cláusulas contractuales involucradas, debido a sus interpretaciones y los numerosos algoritmos de cálcu- lo usuales en estos procesos en organizaciones grandes. Serios contratiempos ocurrieron.

Por ejemplo, en una de las mayores filiales donde hubo problemas con las tres dimensiones señaladas. Una fuerte reacción restrictiva de parte del sindicato de trabajadores a los cambios, tanto de forma como de fondo, contemplados en este PDSI ocasionó el rechazo y aplazamiento del desarrollo e implantación de estos SI durante años. Otra situación se presentó con el SI de costos estándar, donde malas especificaciones dictadas por un UFR no calificado, ocasionaron resistencia al uso del SI por parte de los UF; esta problemática llevaba tiempo vigente al momento de realizar esta investigación.

Adicionalmente, se obtuvo testimonio sobre algunas situaciones muy perju- 
diciales y muy comunes en proyectos de $\mathrm{SI}$, producto de lo anterior, como la delegación en los analistas de informática la atribución de dictar especificaciones funcionales, no tecnológicas, de un SI, una fuente casi segura de posterior rechazo por los UF. En otros casos, el equipo del PDSI fue decepcionado por UFR considerados expertos en su área al dictar especificaciones erradas, dando origen a reprocesos, consumo excesivo de recursos y rechazo al SI una vez terminado.

En este mismo orden, otra situación muy frecuente y costosa cuando se trata de paquetes empresariales tipo ERP, y encontrado también aquí, fue el deficiente apoyo del proveedor del SAP/R3, siendo necesario entrenar al personal interno de analistas y programadores para tomar control del proyecto, generando igualmente serios tropiezos al proceso y costosos incrementos en tiempo y dinero. No pocas veces, el personal de proveedores de ERPs, enviados donde los clientes como expertos, resultan ser informáticos noveles en el dominio del software.

En resumen, en las unidades usuarias donde ocurrieron situaciones irregulares como las anteriores, los desarrollos sufrieron significativos contratiempos en los planes y la satisfacción con los SI involucrados, una vez implementados, alcanzó niveles muy bajos. Lo contrario ocurrió con los otros módulos del SAP/R3 en cuyas áreas usuarias los factores analizados fueron de "calidad".

Un hallazgo significativo reveló que los conocimientos de los UFR sobre las $\mathrm{TI}$, en lo cual la mayoría de los autores basan principalmente su análisis de este constructo, resultaron ser irrelevantes.
Ello confirma lo argumentado sobre la importancia de los conocimientos de los UFR sobre los aspectos funcionales y socio-políticos de la organización y de sus unidades. Finalmente, un resultado práctico para gerentes y practicantes es la lista comprehensiva constituida por las variables indicadoras, como parámetros de control a fin de tomar medidas preventivas. Así, los enfoques cuantitativo y cualitativo arrojaron información sobre el alto nivel del impacto esperado de los factores estudiados y sus consecuencias, encontrándose coincidencia y complementariedad en sus resultados, mostrando también la misma direccionalidad y concordancia con la teoría encontrada.

Ha de resaltarse el rol participativo y protagónico de los UF a lo largo del proceso de desarrollo en este macro PDSI; especialmente en la definición de la funcionalidad necesaria. Por tanto, no se trata de uno de los casos donde las características técnicas y la funcionalidad de un SI adquirido de terceros le son impuestas a los UF.

\section{Conclusiones}

La prueba estadística del modelo, mediante el método PLS, demostró una significativa correlación entre los factores de los UFR analizados y la satisfacción percibida de los mismos UF con sus respectivos SI, como sinónimo de éxito/fracaso. Ello da respuesta a la primera pregunta y al primer objetivo de esta investigación. Igualmente, la respuesta a la segunda pregunta y al segundo objetivo se halla, básicamente, en el análisis hermenéutico/dialéctico. Las principales conclusiones son las siguientes. 
Existe aún un serio reto gerencial respecto a lograr internalizar y "gestionar" adecuadamente el rol de los UFR, cuya (in)capacidad usualmente dará origen a SI (dis)funcionales causantes de (in)satisfacción en ellos mismos y sus representados, los UF, lo cual será el mejor indicador del éxito/fracaso de los SI. De los resultados y de lo visto en la praxis es deducible que en estas dimensiones de los UFR, por sí solas, pueda yacer buena parte de la explicación a la problemática planteada, con especial referencia a las recurrentes pérdidas en las inversiones en SI. Es posible encontrar en los constructos analizados la influencia y el poder ejercible por los UF en las organizaciones. La adecuada selección de los UFR y su exigible responsabilidad continúan sin recibir de la teoría, la investigación y la práctica la atención requerida.

Respecto a las aptitudes y actitudes de los UF aquí analizadas, su evaluación debe ir más allá de las intenciones iniciales de aceptación y uso de los TAMs, antes de ser asignados a un PDSI. Igualmente, se requiere de una planificación previa para adaptar el capital humano y la gestión a los desarrollos tecnológicos. Las referidas estadísticas sobre fracasos parciales y totales continúan con tendencia a agravarse y son los factores socio-conductuales y no los tecnológicos los más preocupantes. Se concluye, finalmente, que aunque el rol de los UF haya sido tratado anteriormente, la problemática estudiada aún persiste a nivel global indicando la necesidad de su profundización mediante enfoques diferentes, como el presente.

Respecto a los aportes adicionales planteados al inicio, la contribución prác- tica comienza con el llamado de alerta y concientización sobre la criticidad de la problemática planteada y continúa con los aspectos analizados sobre los UFR, como parámetros de evaluación para prevenir los riesgos asociados a estas. Además, las variables independientes del modelo propuesto pueden utilizarse para expandir retrospectivamente el reconocido modelo de DeLone y McLean (2003), colocándolas como variables predictivas de sus constructos "calidad de la información" y "calidad del SI" como herramienta de tecnología.

Un hallazgo inesperado, y contrario a lo propuesto por muchas investigaciones, fue la poca relevancia de los conocimientos de los UFR de las $\mathrm{TI}$, durante un PDSI. También lo fue el gran poder social y político capaz de ser ejercido por grupos de trabajadores dentro de organizaciones públicas de grandes dimensiones.

Una sugerencia clave es la de protegerse contractualmente contra fallas del lado de los proveedores de SI, en especial de paquetes complejos como los ERP. Serios aspectos contractuales, los cuales también suelen ser subestimados en estos casos, deben ser definidos lo más detalladamente posible. Sobre todo, en lo referente a la calidad de su mano de obra, costos, tiempos y penalidades en caso de incumplimiento. Junto con la prelación del factor "conocimientos" y los factores conductuales de los UFR, se sugiere como investigaciones futuras la profundización respecto a la gestión del conocimiento y la inteligencia emocional en las organizaciones, con el apoyo de las ciencias de la conducta.

Ciertas limitaciones de este estudio pueden estar en el hecho de haberse ba- 
sado en el PDSI de un software ERP en organizaciones grandes y complejas donde se requirió realizar desarrollos internos de varios de sus módulos, debido a funcionalidad no provista por los SI del ERP, algo muy común en organizaciones con estas características. Otra limitación pudiese hallarse en la realización del estudio en Latinoamérica, en un país en vías de desarrollo. Sin embargo, la problemática analizada no suele ser extraña para muchos PDSI en organizaciones a nivel global, indistintamente de su tamaño o tipo de SI, estimando por tanto que los resultados, conclusiones y sugerencias de este estudio tienen un cierto grado de generalización.

Dado el carácter transversal o sincrónico del estudio, se sugiere para futuras investigaciones adoptar un enfoque diacrónico o longitudinal, a fin de comparar etapas posteriores a la implementación de los SI.

\section{Referencias bibliográficas}

Al-Ahmad, Walid; Al-Fagih, Khalid; Khanfar, Khalid; Alsamara, Khalid; Abuleil, Saleem y Abu-Salem, Hani (2009). A taxonomy of an IT project failure: Root Causes. International Management Review, Vol. 5, № 1, pp. 93-104.

Andrade, Jesús Alberto y Mandrillo, Cósimo (2004). El outsourcing de los sistemas de información en las organizaciones públicas. Revista Venezolana de Gerencia, Vol. 9, № 28, pp.607-622.

Andrade, Jesús Alberto (2003). Tecnologías y sistemas de información en la gestión de conocimiento en las organizaciones. Revista Venezolana de Gerencia, Vol. 8, No 24 , pp. 1-20.
Bacon, Donald; Sauer, Paul y Young, Murray (1995). Composite Reliability in Structural Equations Modelling. Educational and Psychological Measurement, Vol. 55, № 3, pp. 394-406.

Barki, Henri. y Hartwick, Jon (1989). Rethinking the Concept of User Involvement. MIS Quarterly, Vol. 13, No 1, pp. 5363.

Barki, Henri; Rivard, Suzanne y Talbot, Jean (2001). An Integrative Contingency Model of Software Project Risk Management. Journal of Management Information Systems, Vol. 17, № 4, pp. 37-69.

Bartis, Eszter y Mitev, Nathalie (2008). A multiple narrative approach to information systems failure: a successful system that failed. European Journal of Information Systems, Vol. 17, № 2, pp. 112-124.

Bruque, Sebastián; Hernández, $M^{a}$ Jesús y Vargas Alfonso (2004). Condicionantes humanos y de gestión en la implantación y desarrollo de las tecnologías de la información y de la comunicación. Una aplicación al sector de distribución farmacéutica. Dirección y Organización, №30, pp. 89-110.

Byrd, Terry; Thrasher, Evelyn; Lang, Teresa y Davidson, Nancy (2006). A processoriented perspective of IS success: examining the impact of IS on operational cost. Omega, Vol. 34, № 5, pp. 448-460.

Cepeda, Gabriel y Roldán, José Luis (2004). Aplicando la técnica PLS en la administración de empresas. Conocimiento y Competitividad, Congreso ACEDE, Vol. 14, № 14, pp. 74-78.

Chen, Charlie; Chen, Houn-Gee y Yu-Chih Liu, Julie (2011). Discriminative effect of user influence and user responsibility on information system development processes and project management. 
Information and Software Technology, Vol. 53, N² 2, pp. 149-158.

Conboy, Kieran (2010). Project failure en masse: a study of loose budgetary control in ISD projects. European Journal of Information Systems, Vol. 19, № 3, pp. 273-287.

Davis, Fred; Bagozzi, Richard y Warshaw, Paul (1989). User acceptance of computer technology: a comparison of two theoretical models. Management Science, Vol. 35, № 8, pp. 982-1003.

DeLone, William y McLean, Ephraim (2003). The DeLone y McLean model of information systems success: A ten-year update. Journal of Management Information Systems, Vol. 19, $N^{\circ} 4$, pp. 9-30.

García, Gustavo y Rangel, Johanna (2001). Resistencia al cambio tecnológico en las organizaciones durante el desarrollo de sistemas de información para el área de recursos humanos. Revista sobre Relaciones Industriales y Laborales, $N^{\circ} 37$, pp. 69-90.

Goldfinch, Shaun (2007). Pessimism, computer failure, and information systems development in the public sector. Public Administration Review, 67(5), pp. 917-929.

Haenlein, Michael y Kaplan, Andreas (2004). A beginner's guide to partial least squares analysis. Understanding Statistics, Vol. 3, № 4, pp. 283-297.

Hsu Jenny; Huang, Chen-Mei y Hsu, Ping-Yu (2006). The exploration of top management support to the ERP project then influence user satisfaction - use the information, system, service quality, as the moderators. In Proceedings of the Fourth Workshop on Knowledge Economy and Electronic Commerce, Kaohsiung, Taiwan, pp.142-154.

Kappelman, Leon; Mckeeman, Robert y Zhang, Lixuan (2006). Early warning signs of
TI project failure: the dominant dozen. Information Systems Management, Vol. 23, N 4, pp. 31-36.

Kautz, Karlheinz; Dawson, Linda; Nielsen Peter y Russo, Nancy (2010). New trends in information systems development. Information Systems Journal, CFP for Special Issues. http://www.isj-editors.org/? $\quad \mathrm{p}=232$. [Acceso: 10-07-2011].

Laudon, Kenneth y Laudon, Jane (2012). Management Information Systems. Managing the Digital Firm. Prentice Hall: USA.

Lesca, Nicolás. y Caron-Fasan, MarieLaurence (2008). Strategic scanning project failure and abandonment factors: lessons learned. European Journal of Information Systems, Vol. 17, N 4, pp. 371-386.

Llorens, Juan (2005). Gerencia de proyectos de tecnología de información. Los Libros de El Nacional, Colección Minerva. Editorial CEC, SA, Caracas.

Lohmoller, Jan-Bernd (1989). Latent variable Path Modeling with Partial Least Squares. New York: Springer-Verlag.

Markus, M Lynne y Mao, Ji-Ye (2004). Participation in development and implementation -updating an old, tired concept for today's IS contexts. Journal of the Association for Information Systems, Vol. 5, № 11, pp. 514-544.

Meissonier, Régis y Houzé, Emmanuel (2010). Toward an 'IT Conflict-Resistance Theory': action research during IT pre-implementation. European Journal of Information Systems, Vol. 19, $\mathrm{N}^{\circ} 5$, pp. 540-561.

Mithas, Sunil; Tafti, Ali; Bardhan, Indranil y Goh Lie-Mein (2012). Information Technology and firm profitability: mechanisms and empirical evidence. MIS Quarterly, Vol. 36, № 1, pp. 205-224.

Myers, Michael (2009). Qualitative Research in Business and Management, $1^{\text {st }}$. 
edition Cromwell Press Ltd.: Townbridge.

Nonaka, Ikujiro (1991). The knowledge creating company. Harvard Business Review, Vol. 69, № 7, pp. 96-104.

O'hEocha, Colm; Conboy, Kieran y Wang, Xiaofeng (2010). Using Focus Groups in Studies of ISD Team Behaviour. The Electronic Journal of Business Research Methods, Vol. 8, № 2, pp. 119-131.

Ouadahi, Jamal (2008). A qualitative analysis of factors associated with user acceptance and rejection of a new workplace information system in the public sector: A conceptual model. Canadian Journal of Administrative Sciences, Vol. 25, $\mathrm{N}^{\circ} 3, \mathrm{pp}$. 201-213.

Pan, Gary; Hackney, Ray y Pan, Shan (2008). Information Systems implementation failure: Insights from prism. International Journal of Information Management, Vol. 28, № 4, pp. 259-269.

Rainer, Austen y Hall, Tracy (2003). A quantitative and qualitative analysis of factors afecting software processes. The Journal of Systems and Software, Vol. 66, No 1, pp. 7-21.

Ramírez-Correa, Patricio; Roldán-Cataluña, Francisco y Arenas-Gaitán, Jorge (2010). Influencia del Género en la Percepción y Adopción de e-Learning: Estudio Exploratorio en una Universidad Chilena. Journal of Technology Management and Innovation, Vol. 5, № 3, pp. 130-141.

Rastrollo, María Ángeles y Castillo, Ana María (2004). Nuevas TIC y estructura organizativa: de la burocracia vertical a la empresa red. Dirección y Organización, Nº 30, pp. 135-144.

Ravichandran, Thiagarajan y Rai, Arun (2000). Total quality management in information systems development: Key constructs and relationships. Journal of
Management Information Systems, Vol. 16, N³, pp. 119-155.

Saarinen, Timo y Saaksjarvi, Markku (1990). The missing concepts of user participation: an empirical assessment of user participation and information system success. Scandinavian Journal of Information Systems, Vol. 2, No 1 , pp. 25-42.

Saarinen, Timo (1996). An expanded instrument for evaluating information systems success. Information y Management, Vol. 31, № 2, pp. 103-118.

Schultze, Ulrike y Leidner, Dorothy (2002). Studying knowledge management in information systems research: Discourses and theoretical assumptions. MIS Quarterly, Vol. 26, №3, pp.213242.

Shih-Chieh, Jack; Chan, Chien-Lung; Yu-Chih, Julie y Chen, Houn-Gee (2008). The impacts of user review on software responsiveness: Moderating requirements uncertainty. Information y Management, Vol. 45, № 4, pp. 203210.

Somers, Toni; Nelson, Klara y Karimi, Jahangir (2003). Confirmatory factor analysis of the end-user computing satisfaction instrument: replication within an ERP domain. Decision Sciences, Vol. 34, $N^{\circ} 3$, pp. 595-621.

Subramanian, Girish; Jiang, James y Klein, Gary (2007). Software quality and IS project performance improvements from software development process maturity and IS implementation strategies. The Journal of Systems and Software, Vol. 80, N 4, pp. 616-627.

Terry, Julian y Standing, Craig (2004). The value of user participation in eCommerce systems development. Informing Science Journal. Vol. 7, pp. 31-45.

Wagner, Erika y Newell, Sue (2007). Exploring the importance of participation in the 
post implementation period of an ES project: a neglected area. Journal of the Association for Information Systems, Vol. 8, № 10, pp. 508-524.

Walsham, Geoff (1995). Interpretive case studies in IS research: nature and method. European Journal of Information Systems, Vol. 4, № 2, pp. 74-81.

Wold, Herman (1982). Soft modeling: the basic design and some extensions. In K.G. Jöreskog y $\mathrm{H}$. Wold (Eds.) Systems under indirect observations: Causality, structure, prediction. Part 2, North-Holland Press: Amsterdam, pp.1-54.

Wu, Chun-Hui y Fang, Kwoting (2007). The Impact of Organizational Learning on Lack of Team's Expertise Risk in Information Systems Projects. In Proceedings of the IEEE International
Conference on e-Business Engineering, pp.738-743, Hong Kong, China, October 24-26, 2007.

Yin, Robert (1989). Case study research: Design and methods (Rev. ed.). Sage Publishing: California.

Yoruk, Süleyman y Ercan, Sami (2006). Service Quality of Information Systems. In Proceedings of the International Conference on Service Systems and Service Management, pp. 2527, Troyes, France. October 25-27, 2006. IEEE Xplore, digital library. v.II.

Yu-Chih, Julie; Houn-Gee, Henry; Jiang, James y Klein, Gary (2010). Task completion competency and project management performance: The influence of control and user contribution. International Journal of Project Management, Vol. 28, N ${ }^{\circ} 3$, pp. 220-227. 